tion of mutational revertants or contaminating "normal" cells because the treated T4-2 cells could be trypsinized, propagated in monolayer culture and then re-cultured in the three-dimensional system in the absence of antibody, wherein they resumed their "malignant" phenotype. The experiment could be repeated sequentially, thus demonstrating the retention of phenotypic plasticity against an apparently constant genetic background.

The reciprocal phenomenon, the switching of $\mathrm{S} 1$ cells to the "malignant" phenotype of the T4-2 cells, was also demonstrated when function-altering $\beta 4$-integrins or $\alpha 6$-integrin Mabs were applied in a similar protocol. Interestingly, when suspensions of T4-2 cells were treated with the inhibitory $\beta 1$-integrin $\mathrm{Mab}$, their tumorigenicity was reduced in vivo.

Functional redundancy, the involvement of many genes in a single pathway, and oncogene cooperation ${ }^{8}$ make the relationship between genotype and phenotype complex. The lack of a clear association between particular genetic changes and benign breast lesions is, therefore, no argument against clonal expansion models. But, in the absence of explanations of phenotypic advantage and because many genetic alterations may result from, rather than cause, malignant progression, the results of Bissell, Petersen, and colleagues may provoke a more cosmopolitan view of breast tumorigenesis in which interactions between cell and microenvironment play an explicit role.

1. Weaver, V.M. et al. 1997. J. Cell Biol. 137:231-246.

2. Fearon, E. and Vogelstein, B. 1990. Cell 61:759-767.

3. Smith, H.S. et al. 1993. J. Cellular Biochem. 17G:144-152.

4. Steeg, P.S. et al. 1996. Am. J. Pathol. 149:733-738.

5. Holt, J.T. et al. 1996. Nature Genet. 12:298-302.

6. Sharan, S.K. et al. 1997. Nature 386:804-810.

7. Kasami, M. et al. 1997. Am. J. Pathol. 150:in press.

8. Lloyd, A.C. et al. 1997. Genes Dev. 11:663-677.

\title{
Cancer researchers validate and pursue telomerase
}

\section{Debra Robertson}

An overwhelming body of evidence presented by researchers at the 88 th annual meeting of the American Association of Cancer Research* confirms that telomerase is upregulated or reactivated as part of the cancer process. Research on the ribonucleoprotein was the subject of over 150 presentations at the 5-day meeting, up from 30 presented in 1995. "Telomerase is one of the most exciting things I've seen in the cancer field," says Donald Coffey, president of the AACR. "We don't know if this will be a panacea, or not, but it is an Achilles' heel we need to look at."

The catalytic activity of telomerase stabilizes the telomeres by adding hexameric repeats to the ends of the chromosomes in vertebrate germ cells and in the early stage embryo and is inactivated or repressed in all somatic cells except neoplasms.

Studies presented at the San Diego meeting demonstrate that telomerase is expressed in most tumor tissues and occasionally in tissues adjacent to the tumor, possibly representing early metastasis. "Telomerase is found in more than $85 \%$ of all advanced cancers," says Jerry Shay from The University of Texas Southwestern Medical Center in Dallas. "In some types of cancer, such as thyroid, pancreatic, and breast cancer, it is found in closer to $100 \%$ of the cases."

Shay says that in neuroblastomas, the most common solid tumor in children, early stage ( 1 and 2) cancer cells produce much less telomerase than stage 3 or 4 cells, and that this correlates with other genetic markers and outcomes, including survival rate.

Eiso Hiyama, a cancer researcher at the Hiroshima University Medical Center in Japan, using tissue obtained from an endoscopic biopsy of pancreatic ducts, demonstrated that telomerase is an independent marker

Debra Robertson is a freelance writer working in San Diego. for cancer by detecting the enzyme in 12 out of 13 patients in whom cancer was found after abdominal surgery. Patients without evidence of telomerase turned out to be cancer free.

When used in conjunction with histopathology, telomerase improves the identification of hard-to-detect cancers. Louis Dubeau, a researcher at the Kenneth Norris Jr. Comprehensive Cancer Center (Los Angeles, CA) uses telomerase to monitor patients treated for ovarian cancer, a hard-to-

\section{Despite the advances in the use of telomerase as a diagnostic tool, the structure of the holo- enzyme remains elusive.}

treat cancer can spread to the abdominal cavity. Dubeau and his colleagues were able to diagnosis stage one ovarian cancer that is not easily detected by conventional histopathology. "After surgery and chemotherapy, doctors typically perform a major operation to scan the abdomen for signs of cancer," says Dubeau. "Looking for telomerase eliminates the need for the second operation."

The detection of telomerase in cancer offers a solution to oncologists wrestling with the issue of how to determine the effectiveness of traditional cancer treatment (chemotherapy and radiation therapy) and how to better predict probability of disease relapse. "Telomerase is not only important in the diagnosis and prognosis of cancer but it is an excellent marker for residual disease because it is not in normal tissues," says Shay.

Another highlight of the cancer meeting was the development of a potential animal model for telomerase. Ronald DePinho of the Albert Einstein School of Medicine (New York) reported the creation of a telornerase knockout mouse strain. Phenotypically, the absence of telomerase does not have much of an impact on the ability of cells to be transformed by oncogenes, nor is there an effect on the health and well being of the mice in the early generations. "It remains to be determined if this model will be useful with respect to answering questions about cancer and the homeostasis of organs with high renewal potential," says DePinho. "We hope to develop this model so that we can access the role of telomerase in cancer pathogenesis and utilize the system to examine telomerase inhibitors in the prevention or attenuation of cancer."

Despite the advances in the use of telomerase as a diagnostic tool, the structure of the holoenzyme remains elusive. Although the RNA component of the telomerase ribonucleoprotein is cloned in several organism including humans, the protein components that confer catalytic activity remain unidentified. Several groups described the isolation of telomerase-associated proteins but a recent publication in Science $(276: 561-567)$ by Thomas Cech's group at the University of Colorado (Boulder, $\mathrm{CO}$ ) reports the purification of a $123 \mathrm{kDa}$ (p123) protein from Euplotes aediculatus, a ciliated protozoan, and from Saccharomyces cerevisiae that contains reverse transcriptase motifs. This motif is consistent with the catalytic activity of telomerase, which works like a reverse transcriptase by binding the end of the chromosome and synthesizing DNA. "The race is on to find the equivalent protein in humans," predicts Shay. "Once the holoenzyme is cloned then the catalytic subunit, the RNA, and potentially the proteins that bind and stabilize telomeres can be targets for cancer therapy.

* San Diego, CA, April 12-16, 1997. 